\title{
Basal DNA repair machinery is subject to positive selection in ionizing-radiation-resistant bacteria Haïtham Sghaier* ${ }^{* 1}$, Kaïs Ghedira ${ }^{\dagger 2}$, Alia Benkahla² and Insaf Barkallah ${ }^{1}$
}

Address: ${ }^{1}$ Unit of Microbiology and Molecular Biology, National Center for Nuclear Sciences and Technologies (CNSTN), Sidi Thabet Technopark, 2020 Sidi Thabet, Tunisia and ${ }^{2}$ Group of Bioinformatics and Modelling, Laboratory of Immunology, Vaccinology, and Molecular Genetics, Institute Pasteur of Tunis, 13, place Pasteur BP 74, 1002 Tunis, Tunisia

Email: Haïtham Sghaier* - sghaier.haitham@gmail.com; Kaïs Ghedira - kaisghedira@yahoo.fr; Alia Benkahla - Alia.Benkahla@pasteur.rns.tn; Insaf Barkallah - I.BARKALLAH@cnstn.rnrt.tn

* Corresponding author †Equal contributors

Published: 21 June 2008

BMC Genomics 2008, 9:297 doi:10.1 I86/|47|-2164-9-297
Received: 15 December 2007

Accepted: 21 June 2008

This article is available from: http://www.biomedcentral.com/I47I-2/64/9/297

(C) 2008 Sghaier et al; licensee BioMed Central Ltd.

This is an Open Access article distributed under the terms of the Creative Commons Attribution License (http://creativecommons.org/licenses/by/2.0), which permits unrestricted use, distribution, and reproduction in any medium, provided the original work is properly cited.

\begin{abstract}
Background: lonizing-radiation-resistant bacteria (IRRB) show a surprising capacity for adaptation to ionizing radiation and desiccation. Positive Darwinian selection is expected to play an important role in this trait, but no data are currently available regarding the role of positive adaptive selection in resistance to ionizing-radiation and tolerance of desiccation. We analyzed the four known genome sequences of IRRB (Deinococcus geothermalis, Deinococcus radiodurans, Kineococcus radiotolerans, and Rubrobacter xylanophilus) to determine the role of positive Darwinian selection in the evolution of resistance to ionizing radiation and tolerance of desiccation.

Results: We used the programs MultiParanoid and DnaSP to deduce the sets of orthologs that potentially evolved due to positive Darwinian selection in IRRB. We find that positive selection targets 689 ortholog sets of IRRB. Among these, 58 ortholog sets are absent in ionizing-radiationsensitive bacteria (IRSB: Escherichia coli and Thermus thermophilus). The most striking finding is that all basal DNA repair genes in IRRB, unlike many of their orthologs in IRSB, are subject to positive selection.

Conclusion: Our results provide the first in silico prediction of positively selected genes with potential roles in the molecular basis of resistance to $\gamma$-radiation and tolerance of desiccation in IRRB. Identification of these genes provides a basis for future experimental work aimed at understanding the metabolic networks in which they participate.
\end{abstract}

\section{Background}

In this paper, we consider ionizing-radiation-resistant bacteria (IRRB) as "non-spore-forming bacteria that can protect their cytosolic proteins from oxidation and tolerate many DNA double-strand breaks (DSBs) after exposure to high, acute ionizing radiation (dose greater than 1 kilogray (kGy) for $90 \%$ reduction $\left(\mathrm{D}_{10}\right)$ in Colony Forming Units (CFUs)), and can resist prolonged desiccation."
Over the past five decades, Deinococcus radiodurans $\left(\mathrm{D}_{10} \approx\right.$ $15 \mathrm{kGy}$ ) has been a model for understanding many of the basic principles that govern resistance to ionizing radiation and tolerance of desiccation (for review see [1]). Recent findings indicated that the mutual nature of $D$. radiodurans's $\gamma$-radiation resistance and desiccation tolerance resides in cytosolic Mn-dependent mechanisms that protect proteins from oxidative modifications that intro- 
duce carbonyl groups $[2,3]$. Like most IRRB, D. radiodurans accumulates about 300 times more $\mathrm{Mn}$ (II) than ionizing-radiation-sensitive bacteria (IRSB) [4] such as Escherichia coli $\left(\mathrm{D}_{10} \approx 0.7 \mathrm{kGy}\right)$ and Thermus thermophilus $\left(\mathrm{D}_{10} \approx 0.8 \mathrm{kGy}\right)[5,6]$. Due to its high concentration of intracellular Mn(II) ions and the consequent protection of proteins, $D$. radiodurans can survive $10 \mathrm{kGy}$ of ionizing radiation, a dose that causes approximately 100 DNA DSBs per genome. This species can also survive 8 days in a desiccator with no obvious DNA DSBs $[2-4,7,8]$. Early on, analysis of $D$. radiodurans's transcriptional response revealed that the cellular responses to ionizing radiation and desiccation exhibit substantial overlap [9]. In this context, 41 ionizing-radiation-sensitive strains of $D$. radiodurans were also shown to be sensitive to desiccation [7]. Furthermore, the mutational inactivation of the genes DR_1172 - encodes a homolog of LEA76, a group 3 LEA (late embryogenesis abundant) protein - and DR_B0118 - encodes a protein that is expressed during dehydration by the resurrection plant, Craterostigma plantagineum greatly sensitized $D$. radiodurans to desiccation, but not to ionizing radiation [10]. The former result based on a collection of ionizing-radiation-sensitive strains of $D$. radiodurans is consistent with evidence that dried bacterial cells exhibit a substantial number of DNA DSBs, single-strand breaks, and DNA crosslinks [11], DNA damage that is also observed following exposure to ionizing radiation [12]. Indeed, ionizing radiation and desiccation introduce similar types of DNA damage - DNA DSBs - in D. radiodurans [7]. The latter result based on the mutational inactivation of the genes DR_1172 and DR_B0118 considerably strengthens the hypothesis that the $D$. radiodurans's desiccation tolerance could be a consequence of this organism's adaptation to ionizing radiation (radiation adaptation hypothesis), particularly that the origin of ionizing-radiation resistance in bacteria can be explained as an adaptation to environmental radiation [13]. For example, Deinococcus sp. have been isolated from the subsurfaces of hydrothermal vents at depths of 64.8-128.9 m below the sea floor (mbsf) [14]. D'Hondt and colleagues [15] have recently surveyed environments representative of a broad range of subsurface conditions that can be found in marine sediments. Among the most striking features of deeply buried sediments (20-100 mbsf) are Mnrich sites with high natural $\gamma$-radiation levels. Ionizingradiation levels in such deposits might have been much higher on ancient Earth (Daly MJ, personal communication, 2006). Concerning the close relationship between bacterial ionizing-radiation resistance and desiccation tolerance $[2,3,7,9]$, many of the environments from which IRRB have been isolated can be considered to be dry, and it has been shown that many of these strains are also desiccation tolerant [16]. Moreover, it was demonstrated that IRRB are present at higher numbers in an arid soil than in a nonarid soil, and that IRRB in the arid soil are recovered after exposure to higher doses than the doses which allow recovery of bacteria from the nonarid soil [16]. In this context, many IRRB (i.e. Deinococcus deserti, Deinococcus sonorensis, etc.) have been isolated from the desert $[16,17]$, indicating they have the capacity to adapt to harsh environments.

Three principal mechanisms may have contributed to the adaptability of IRRB: positive Darwinian selection, lateral gene transfer, and gene regulation [18]. Previous studies have discussed the importance of lateral gene transfer and gene regulation in Deinococcus evolution [5,6]. However, no previous research has addressed the role of positive Darwinian selection in resistance to ionizing-radiation and tolerance of desiccation in IRRB. In this paper, we assess the role of positive Darwinian selection by analysis of the genomes of IRRB. Among the many IRRB, completely sequenced genomes are only available for $D$. geothermalis $\left(\mathrm{D}_{10} \approx 15 \mathrm{kGy}\right), D$. radiodurans, Kineococcus radiotolerans $\left(\mathrm{D}_{10} \approx 2 \mathrm{kGy}\right)$, and Rubrobacter xylanophilus $\left(D_{10} \approx 5.5 \mathrm{kGy}\right)[1,6,19,20]$. When bacterial species of the genera Deinococcus (D. geothermalis and D. radiodurans) and Kineococcus (K. radiotolerans) were tested in vitro for desiccation tolerance, all were found to be tolerant to desiccation $[4,21]$. We are not aware of any studies that examined R. xylanophilus for desiccation tolerance, but since (1) many IRRB isolated from the desert - they are one way or another tolerant to desiccation - cluster within the monophyletic Rubrobacteria subgroup which is specialized for particular niches present in arid soils [22,23], (2) bacterial species of the genus Rubrobacter ( $R$. radiotolerans, $R$. taiwanensis, and R. xylanophilus) belong to IRRB $[24,25]$, and (3) there has to our knowledge never been a report of desiccation-sensitive IRRB, we included $R$. xylanophilus in our study.

Positive Darwinian selection leads to the fixation of advantageous mutations and is the fundamental process behind adaptive changes in genes (reference [26] and citations therein). Many statistical methods have been developed for detecting adaptive molecular evolution [27-29], such as the "conservative" PAML program $[30,31]$. In this report, we used a freely available software package with a user-computer interface (DnaSP) [32] to identify genes under positive selection in IRRB. This program calculates the ratios of nonsynonymous to synonymous mutation rates $(\mathrm{Ka} / \mathrm{Ks})$ in protein coding genes. The $\mathrm{Ka} / \mathrm{Ks}$ ratio measures the strength of selection, with values $>1$ indicative of positive selection [29].

\section{Results and discussion}

At the time we began this work, the GOLD database [20] documented four completely sequenced IRRB genomes: D. geothermalis DSM 11300 [6], D. radiodurans $\mathrm{R}_{1}$ [19], $K$. radiotolerans SRS30216 [21], and R. xylanophilus DSM 
9941 [24]. We identified 734 orthologs that were present in all four species. To validate our subsequent results, we deduced hits in IRSB genomes (Escherichia coli 536, Escherichia coli K-12, Thermus thermophilus HB8, and Thermus thermophilus HB27) of the 734 ortholog sets in IRRB [see Additional file 1]. Then, we used DnaSP to examine the aligned ortholog sets among IRRB [see Additional files 2, 3, 4, 5, 6, 7, 8] and IRSB [see Additional files 9, 10, 11] to search for evidence of positive selection [32]. We identified 689 ortholog sets among IRRB as potentially subject to positive Darwinian selection (Additional file 1, genes in bold). Supporting our conclusion that these genes have been under selective pressure from niches with high natural $\gamma$-radiation or desiccation levels [13], we found that many of these positively selected genes are involved in resistance to $\gamma$-radiation resistance and tolerance of desiccation $[6,9]$. To find out if there were any biais due to phylogenetic relationships, patterns of positive selection in Deinococcus and Thermus species were compared, because phylogenetically the closest relative of Deinococcus is the genus Thermus [5]. As patterns of positive selection in Deinococcus and Thermus species were different [see Additional file 1], we concluded that DnaSP scores are related to resistance to ionizing-radiation and tolerance of desiccation phenotypes and not to the phylogenetic relationships of the species. But it should be noted that our bioinformatical method used to detect positive selection is based on sequence alignments, and therefore we cannot exclude that in some cases we have included false positives because of improper alignments.

We defined two important families of positively selected genes in IRRB: i) Group-I genes, which are under positive selection in IRRB but under neutral or purifying selection in IRSB (125 genes, highlighted in light gray in Additional file 1), and ii) Group-II genes, which are under positive selection in IRRB but absent in all IRSB ( 58 genes, highlighted in dark gray in Additional file 1). These two groups of positively selected genes in IRRB may provide insight into the molecular adaptations to ionizing radiation and desiccation. For instance, Group-II includes DNA repairrelated proteins (DR_0192, MutT/NUDIX family protein; DR_2074, putative 3-methyladenine DNA glycosylase), oxidative damage-related proteins (DR_2242, thiol-specific antioxidant protein; DR_2538, cytochrome P450) and water stress-associated proteins (DR_0463, maltooligosyltrehalose synthase; DR_0464, putative maltooligosyltrehalose trehalohydrolase). These conclusions are in concordance with DNA-centric hypotheses (for example, [33]), protein-centric hypotheses [2,3], and the water replacement with trehalose hypothesis [34] as protection mechanisms against ionizing radiation and desiccation. Our results support the idea that resistance to ionizing radiation and tolerance of desiccation are two complex phenotypes, and suggest that protection and repair mechanisms are complementary in IRRB.

The $D$. radiodurans genome has most of the DNA repair proteins found in E. coli [19]. Broad-based bioinformatic and experimental studies have concluded that $D$. radiodurans uses a relatively conventional set of DNA repair and protection mechanisms, but that these mechanisms are much more efficient than in IRSB $[2,3,9,19]$. We found that some of the accessory DNA repair genes in Deinococcus that are extremely important for resistance phenotypes (e.g., pprI (DR_0167, Dgeo_0395) and pprA (DR_A0346, Dgeo_2628) $[35,36])$ have no orthologs in Kineococcus and Rubrobacter. Similarly, the five transcripts of $D$. radiodurans (ddrA, DR_0423; ddrB, DR_0070; $d d r C$, DR_0003; $d d r D$, DR_0326; pprA, DR_A0346) that are most highly induced following ionizing radiation and recovery from desiccation [9] were not present in Kineococcus and Rubrobacter.

It seems likely that the shared ability of IRRB to survive the damaging effects of ionizing radiation and desiccation is the result of basal DNA repair pathways and that basal DNA repair genes have been acted upon by positive selection. Table 1 shows that, unlike many of their orthologs in IRSB [37], all DNA replication, repair, and recombination genes in IRRB were subject to positive selection. Three major DNA repair genes showed remarkably strong evidence for positive selection: DR_1707 (Ka/Ks = 3.15), DR_0906 $(\mathrm{Ka} / \mathrm{Ks}=3.4)$ and DR_1913 $(\mathrm{Ka} / \mathrm{Ks}=3.48)$.

Zahradka et al. [33] reported the remarkable efficiency of DNA repair enzymes in D. radiodurans during recovery from ionizing radiation and proposed a model named 'Extended Synthesis-Dependent Strand Annealing' (ESDSA). This model proposes that DNA polymerase I (PolA, DR_1707) accounts for the high fidelity of RecAdependent DNA DSB fragment assembly. recA (DR_2340), a central gene to genomic restoration, is upregulated in $D$. radiodurans cultures recovering from ionizing radiation and desiccation [9], and Table 1 shows that recA is positively selected. In particular, PolA of $D$. radiodurans supports very efficient DNA replication at the earliest stages of recovery and is present at higher levels than during normal DNA replication [33]. It is unclear whether polA paralogs participate in ESDSA. However, the presence of polA paralogs that are subject to positive selection (unlike orthologs in IRSB) suggests that these paralogs may be involved in genomic networks of resistance to $\gamma$ radiation and tolerance of desiccation (see Table 1). A positively selected polA paralog set might also explain the results of Gutman et al. [38], who showed that ionizingradiation-sensitive $D$. radiodurans polA mutants are fully complemented by expression of the polA gene from the relatively ionizing-radiation sensitive E. coli. A more com- 
Table I: Replication, repair, and recombination genes under positive selection in ionizing-radiation-resistant bacteria (IRRB)a

\begin{tabular}{|c|c|c|c|}
\hline Orthologs & Function & $(\mathrm{Ka} / \mathrm{Ks})^{\mathrm{b}}$ & D value (Tajima test) ${ }^{\mathrm{b}}$ \\
\hline & DNA polymerase & & \\
\hline DR_0467 Dgeo_1609 Krad_R0056 Rxyl_0486 & Hypothetical DNA polymerase & n.a.c & -1.779 \\
\hline DR_0507 Dgeo_0255 Krad_3I87 Rxyl_1096 & DNA polymerase III, $\alpha$ subunit* & n.a. & -1.725 \\
\hline DR_0856 Dgeo_1818 Krad_3247 Rxyl_2984 & DNA polymerase III, $\varepsilon$ subunit & 2.81 & \\
\hline DR_1244 Dgeo_0745 Krad_3423 Rxyl_I5 I8 & Putative DNA polymerase III, $\delta$ subunit & 1.68 & \\
\hline DR_I707 Dgeo_1666 Krad_295I Rxyl_2025 & DNA-directed DNA polymerase* & 3.15 & \\
\hline DR_I75I Dgeo_I556 Krad_I52I Rxyl_0503 & DNA polymerase-related protein & n.a. & -1.744 \\
\hline DR_24I0 Dgeo_21 35 Krad_R0007 Rxyl_2444 & DNA polymerase III, $\tau / \gamma$ subunit* & 2.3 & \\
\hline
\end{tabular}

\section{Replication complex}

\begin{tabular}{lll}
\hline DR_0100 Dgeo_0165 Krad_4338 Rxyl_0045 & Single-stranded DNA-binding protein* & n.a. \\
DR_0549 Dgeo_2037 Krad_4333 Rxyl_0852 & Replicative DNA helicase & n.a. \\
DR_060I Dgeo_R0043 Krad_336I Rxyl_I502 & DNA primase* & n.a. \\
DR_0906 Dgeo_0546 Krad_0006 Rxyl_0005 & DNA gyrase, subunit B* & -I.539 \\
DR_1374 Dgeo_200I Krad_0487 Rxyl_I964 & DNA topoisomerase I* & 3.4 \\
DR_1913 Dgeo_I016 Krad_0007 Rxyl_0006 & DNA gyrase, subunit A* & 3.13 \\
\end{tabular}

\section{Other DNA-associated proteins}

\begin{tabular}{|c|c|c|c|}
\hline DR_0I20 Dgeo_2345 Krad_I409 Rxyl_I396 & smf protein^ & 1.87 & \\
\hline DR_0289 Dgeo_0248 Krad_0422 Rxyl_2676 & Endonuclease III^ & 2.82 & \\
\hline DR_0440 Dgeo_0327 Krad_3056 Rxyl_I322 & Holliday junction resolvase* & 2.06 & \\
\hline DR_0493 Dgeo_0442 Krad_I377 Rxyl_2433 & Formamidopyrimidine-DNA glycosylase & 2.37 & \\
\hline DR_0596 Dgeo_0404 Krad_3053 Rxyl_1324 & Holliday junction DNA helicase* & n.a. & -1.789 \\
\hline DR_1089 Dgeo_1620 Krad_0004 Rxyl_0004 & RecF protein* & n.a. & -1.342 \\
\hline DR_II05 Dgeo_I2I2 Krad_0603 Rxyl_2178 & DNA repair protein* & 2.61 & \\
\hline DR_1274 Dgeo_0726 Krad_3054 Rxyl_I323 & Holliday junction binding protein^ & 1.88 & \\
\hline DR_1354 Dgeo_II24 Krad_2935 Rxyl_2010 & Excinuclease $A B C$, subunit $C^{*}$ & n.a. & -1.4|| \\
\hline DR_1477 Dgeo_II94 Krad_3I47 Rxyl_|453 & DNA repair protein & 2.08 & \\
\hline DR_I532 Dgeo_0545 Krad_I067 Rxyl_0909 & Transcription-repair coupling factor* & 3.05 & \\
\hline DR_177I Dgeo_0694 Krad_2940 Rxyl_2016 & Excinuclease $A B C$, subunit $A$ & n.a. & -1.678 \\
\hline DR_1775 Dgeo_0868 Krad_0757 Rxyl_0825 & DNA helicase II* & n.a. & -1.450 \\
\hline DR_1916 Dgeo_II39 Krad_I368 Rxyl_137I & DNA helicase RecG* & 3.35 & \\
\hline DR_1921 Dgeo_0824 Krad_2554 Rxyl_0449 & Exonuclease SbcD, putative & 2.36 & \\
\hline DR_1922 Dgeo_0823 Krad_2553 Rxyl_0448 & Exonuclease SbcC & 1.82 & \\
\hline DR_1949 Dgeo_I623 Krad_I405 Rxyl_I394 & Ribonuclease HII^ & n.a. & -1.642 \\
\hline DR_2074 Dgeo_I660 Krad_3 I54 Rxyl_1309 & Putative 3-methyladenine DNA glycosylase* & 3.25 & \\
\hline DR_2275 Dgeo_I890 Krad_2942 Rxyl_202I & Excinuclease $A B C$, subunit $B^{*}$ & n.a. & -1.767 \\
\hline DR_2285 Dgeo_0019 Krad_0599 Rxyl_2229 & A/G-specific adenine glycosylase* & 1.85 & \\
\hline DR_2340 Dgeo_2138 Krad_|492 Rxyl_|423 & RecA protein* & n.a. & -1.179 \\
\hline DR_2584 Dgeo_0107 Krad_4325 Rxyl_1215 & DNA-3-methyladenine glycosidase II, putative* & n.a. & -1.607 \\
\hline
\end{tabular}

aIRRB are Deinococcus geothermalis, Deinococcus radiodurans, Kineococcus radiotolerans and Rubrobacter xylanophilus.

bPositive selection: $(\mathrm{Ka} / \mathrm{Ks})>$ I or Tajima's $\mathrm{D}<0$.

not available. See Methods.

- Genes under positive selection in IRRB and under neutral or purifying selection in ionizing-radiation-sensitive bacteria (IRSB). *Genes under positive selection in both IRRB and IRSB.

$\checkmark$ Genes under positive selection in IRRB for which orthologs were present in some, but not all, IRSB.

* Genes under positive selection in IRRB for which there are no orthologs in all IRSB.

See Methods and Additional file I.

plete examination of these paralogous proteins will be necessary to prove this hypothesis.

Genes encoding the two subunits of the DNA gyrase, DR_1913 (gyrA) and DR_0906 (gyrB), are also induced by ionizing radiation and desiccation [9], and this presumably explains their strong positive selection values. This implies that DNA supercoiling is important for DNA repair following the deleterious effects of ionizing radiation and desiccation. 
Four additional proteins involved in DNA repair are also subject to strong positive selection: (1) DR_1374 (Ka/Ks = 3.13; DNA topoisomerase I, Uniprot Q9RUL0), (2) DR_1532 (Ka/Ks $=3.05$; a transcription-repair coupling factor, Uniprot Q9RU62), (3) DR_1916 (Ka/Ks = 3.35; RecG helicase likely to be involved in DNA DSB repair, Uniprot Q9RT50), and (4) DR_2074 (Ka/Ks = 3.25; putative 3-methyladenine DNA glycosylase, Uniprot Q9RSQ0). This is likely the result of continuous interaction between IRRB and natural niches, which have high levels of $\gamma$-radiation or high probability of desiccation [1317].

The present work has identified the first suite of genes that are under positive Darwinian selection in IRRB. Our results can serve as a useful background to guide future physiological and biochemical experiments examining resistance to ionizing radiation and tolerance of desiccation. As more IRRB genome sequences become available, particularly in more distantly related species, we expect that our methods will provide a sensitive and controlled approach for detection of genes that have been subject to positive Darwinian selection for resistance to ionizing radiation and tolerance of desiccation.

\section{Methods}

To identify genes under positive selection in IRRB, the DnaSP program requires an aligned set of orthologous sequences [32]. Thus, we initiated our analyses by performing comparisons between all fully sequenced IRRB genomes (Deinococcus geothermalis DSM 11300 [6], Deinococcus radiodurans $\mathrm{R}_{1}$ [19], Kineococcus radiotolerans SRS30216 [21], and Rubrobacter xylanophilus DSM 9941 [24]). Information on complete and ongoing IRRB genome sequencing projects was obtained from the GOLD database [20]. Genome sequences were downloaded from the National Center for Biotechnology Information (NCBI) RefSeq repository [39]. For comparison, we used the genomes of 4 IRSB: Escherichia coli 536 (UPEC), Escherichia coli K-12 MG1655, Thermus thermophilus HB8, and Thermus thermophilus HB27 [20,39]. MultiParanoid was used to find orthologous relationships [40]. Orthologous sequences were imported into Bioedit Sequence Alignment Editor (version 7.0.9) [41], and the sequences for each ortholog set were aligned with CLUSTAL W [42]. To test for positive selection, we used DnaSP (version 4.0) [32]. Using the Jukes and Cantor method [43], DnaSP calculates the nonsynonymous (Ka) and synonymous (Ks) substitution rates for each codon. When the nonsynonymous rate was greater than the synonymous rate $(\mathrm{Ka} / \mathrm{Ks}>1)$, this is indicative of positive Darwinian selection [29,32]. When it was not possible to calculate Ka or Ks, we calculated Tajima's D value (in addition to a statistical significance P value), with the confi- dence limit of $\mathrm{D}$ equal to $99.9 \%$. Negative values of Tajima's D indicated positive selection $[27,44]$.

\section{Authors' contributions}

$\mathrm{HS}, \mathrm{KG}, \mathrm{AB}$ and IB designed the study. HS and KG implemented the methods, analyzed the data, and wrote the manuscript. HS and IB analyzed all biological data. KG and $A B$ supervised and oversaw all computational aspects. All authors have read and approved the final version of the manuscript.

\section{Additional material}

\begin{abstract}
Additional file 1
Common genes in ionizing-radiation-resistant bacteria (IRRB) and positive Darwinian selection. Bold letters denote genes under positive selection, whilst non-bold letters denote genes under neutral or purifying selection. Light gray shading indicates genes under positive selection in IRRB and under neutral or purifying selection in IRSB. Dark gray shading indicates genes that are under positive selection in IRRB for which orthologs are absent in all IRSB (see Methods).

Click here for file

[http://www.biomedcentral.com/content/supplementary/1471-

2164-9-297-S1.xls]
\end{abstract}

\section{Additional file 2}

Alignment of sets of orthologs in ionizing-radiation-resistant bacteria (IRRB). Sets of orthologs present in all IRRB were aligned with CLUSTAL $W$ following detection with MultiParanoid (see Methods).

Click here for file

[http://www.biomedcentral.com/content/supplementary/14712164-9-297-S2.exe]

\section{Additional file 3}

Alignment of sets of orthologs in ionizing-radiation-resistant bacteria (IRRB). Sets of orthologs present in all IRRB were aligned with CLUSTAL W following detection with MultiParanoid (see Methods).

Click here for file

[http://www.biomedcentral.com/content/supplementary/14712164-9-297-S3.rar]

\section{Additional file 4}

Alignment of sets of orthologs in ionizing-radiation-resistant bacteria (IRRB). Sets of orthologs present in all IRRB were aligned with CLUSTAL $W$ following detection with MultiParanoid (see Methods).

Click here for file

[http://www.biomedcentral.com/content/supplementary/1471-

2164-9-297-S4.rar]

\section{Additional file 5}

Alignment of sets of orthologs in ionizing-radiation-resistant bacteria (IRRB). Sets of orthologs present in all IRRB were aligned with CLUSTAL $W$ following detection with MultiParanoid (see Methods).

Click here for file

[http://www.biomedcentral.com/content/supplementary/14712164-9-297-S5.rar] 


\section{Additional file 6}

Alignment of sets of orthologs in ionizing-radiation-resistant bacteria (IRRB). Sets of orthologs present in all IRRB were aligned with CLUSTAL W following detection with MultiParanoid (see Methods).

Click here for file

[http://www.biomedcentral.com/content/supplementary/1471-

2164-9-297-S6.rar]

\section{Additional file 7}

Alignment of sets of orthologs in ionizing-radiation-resistant bacteria (IRRB). Sets of orthologs present in all IRRB were aligned with CLUSTAL $W$ following detection with MultiParanoid (see Methods).

Click here for file

[http://www.biomedcentral.com/content/supplementary/14712164-9-297-S7.rar]

\section{Additional file 8}

Alignment of sets of orthologs in ionizing-radiation-resistant bacteria (IRRB). Sets of orthologs present in all IRRB were aligned with CLUSTAL W following detection with MultiParanoid (see Methods).

Click here for file

[http://www.biomedcentral.com/content/supplementary/14712164-9-297-S8.rar]

\section{Additional file 9}

Alignment of sets of orthologs in ionizing-radiation-sensitive bacteria (IRSB) for which there are orthologs in all IRRB. Sets of orthologs present in all IRSB, and for which there are orthologs in all IRRB, were aligned with CLUSTAL W following detection with MultiParanoid (see Methods)

Click here for file

[http://www.biomedcentral.com/content/supplementary/1471-

2164-9-297-S9.exe]

\section{Additional file 10}

Alignment of sets of orthologs in ionizing-radiation-sensitive bacteria (IRSB) for which there are orthologs in all IRRB. Sets of orthologs present in all IRSB, and for which there are orthologs in all IRRB, were aligned with CLUSTAL W following detection with MultiParanoid (see Methods).

Click here for file

[http://www.biomedcentral.com/content/supplementary/14712164-9-297-S10.rar]

\section{Additional file 11}

Alignment of sets of orthologs in ionizing-radiation-sensitive bacteria (IRSB) for which there are orthologs in all IRRB. Sets of orthologs present in all IRSB, and for which there are orthologs in all IRRB, were aligned with CLUSTAL W following detection with MultiParanoid (see Methods).

Click here for file

[http://www.biomedcentral.com/content/supplementary/1471-

2164-9-297-S11.rar]

\section{Acknowledgements}

We thank the two reviewers of this manuscript for very helpful comments. This work was performed under the auspices of the Tunisian National Center for Nuclear Sciences and Technologies (CNSTN) in collaboration with Institute Pasteur (Tunis). The authors are grateful for the assistance of
Sana Fekih (Institute Pasteur, Tunisia), Ahmed Zaki Jenhani (Institute Pasteur, Tunisia), and Issay Narumi (JAEA, Japan).

\section{References}

I. Cox MM, Battista JR: Deinococcus radiodurans - the consummate survivor. Nat Rev Microbiol 2005, 3(I I):882-892.

2. Daly MJ, Gaidamakova EK, Matrosova VY, Vasilenko A, Zhai M, Leapman RD, Lai B, Ravel B, Li SM, Kemner KM, et al.: Protein oxidation implicated as the primary determinant of bacterial radioresistance. PLoS Biol 2007, 5(4):e92.

3. Fredrickson JK, Li SM, Gaidamakova EK, Matrosova VY, Zhai M, Sulloway HM, Scholten JC, Brown MG, Balkwill DL, Daly MJ: Protein oxidation: key to bacterial desiccation resistance? ISME 2008, 2(4):393-403.

4. Daly MJ, Gaidamakova EK, Matrosova VY, Vasilenko A, Zhai M, Venkateswaran A, Hess M, Omelchenko MV, Kostandarithes HM, Makarova KS, et al: Accumulation of Mn(II) in Deinococcus radiodurans facilitates gamma-radiation resistance. Science 2004, 306(5698): $1025-1028$.

5. Omelchenko MV, Wolf YI, Gaidamakova EK, Matrosova VY, Vasilenko A, Zhai M, Daly MJ, Koonin EV, Makarova KS: Comparative genomics of Thermus thermophilus and Deinococcus radiodurans: divergent routes of adaptation to thermophily and radiation resistance. $B M C$ Evol Biol 2005, 5:57.

6. Makarova KS, Omelchenko MV, Gaidamakova EK, Matrosova VY, Vasilenko A, Zhai M, Lapidus A, Copeland A, Kim E, Land M, et al:: Deinococcus geothermalis: The Pool of Extreme Radiation Resistance Genes Shrinks. PLoS ONE 2007, 2(9):e955.

7. Mattimore V, Battista JR: Radioresistance of Deinococcus radiodurans: functions necessary to survive ionizing radiation are also necessary to survive prolonged desiccation. I Bacteriol 1996, I78(3):633-637.

8. Battista JR: Against all odds: the survival strategies of Deinococcus radiodurans. Annu Rev Microbiol 1997, 51:203-224.

9. Tanaka M, Earl AM, Howell HA, Park MJ, Eisen JA, Peterson SN, Battista JR: Analysis of Deinococcus radiodurans 's transcriptional response to ionizing radiation and desiccation reveals novel proteins that contribute to extreme radioresistance. Genetics 2004, 168(1):21-33.

10. Battista JR, Park MJ, McLemore AE: Inactivation of two homologues of proteins presumed to be involved in the desiccation tolerance of plants sensitizes Deinococcus radiodurans $\mathbf{R} \mathbf{I}$ to desiccation. Cryobiology 200I, 43(2): I33-139.

II. Dose K, Bieger-Dose A, Labusch M, Gill M: Survival in extreme dryness and DNA-single-strand breaks. Adv Space Res 1992, I 2(4):22I-229.

12. Ward JF: Molecular mechanisms of radiation-induced damage in nucleic acids. Adv Radiat Biol 1977, 5:181-239.

13. Sghaier H, Narumi I, Satoh K, Ohba H, Mitomo H: Problems with the current deinococcal hypothesis: an alternative theory. Theory Biosci 2007, I 26(1):43-45.

14. Kimura H, Asada R, Masta A, Naganuma T: Distribution of microorganisms in the subsurface of the manus basin hydrothermal vent field in Papua New Guinea. Appl Environ Microbiol 2003 , 69(I):644-648.

15. D'Hondt S, Jorgensen BB, Miller DJ, Batzke A, Blake R, Cragg BA, Cypionka H, Dickens GR, Ferdelman T, Hinrichs KU, et al: Distributions of microbial activities in deep subseafloor sediments. Science 2004, 306(5705):2216-2221.

16. Rainey FA, Ray K, Ferreira M, Gatz BZ, Nobre MF, Bagaley D, Rash BA, Park MJ, Earl AM, Shank NC, et al.: Extensive diversity of ionizing-radiation-resistant bacteria recovered from Sonoran Desert soil and description of nine new species of the genus Deinococcus obtained from a single soil sample. Appl Environ Microbiol 2005, 7 I(9):5225-5235.

17. de Groot A, Chapon V, Servant P, Christen R, Saux MF, Sommer S, Heulin T: Deinococcus deserti sp. nov., a gamma-radiation-tolerant bacterium isolated from the Sahara Desert. Int J Syst Evol Microbiol 2005, 55(Pt 6):244I-2446.

18. Lefebure T, Stanhope MJ: Evolution of the core and pan-genome of Streptococcus: positive selection, recombination, and genome composition. Genome Biol 2007, 8(5):R7I

19. White O, Eisen JA, Heidelberg JF, Hickey EK, Peterson JD, Dodson RJ, Haft DH, Gwinn ML, Nelson WC, Richardson DL, et al.: Genome 
sequence of the radioresistant bacterium Deinococcus radiodurans RI. Science 1999, 286(5444): I57I-I 577.

20. Liolios K, Mavromatis K, Tavernarakis N, Kyrpides NC: The Genomes On Line Database (GOLD) in 2007: status of genomic and metagenomic projects and their associated metadata. Nucleic Acids Res 2008:D475-479.

21. Phillips RW, Wiegel J, Berry CJ, Fliermans C, Peacock AD, White DC, Shimkets LJ: Kineococcus radiotolerans sp. nov., a radiationresistant, gram-positive bacterium. Int J Syst Evol Microbiol 2002, 52(Pt 3): $933-938$

22. Chanal A, Chapon V, Benzerara K, Barakat M, Christen R, Achouak W, Barras F, Heulin T: The desert of Tataouine: an extreme environment that hosts a wide diversity of microorganisms and radiotolerant bacteria. Environ Microbiol 2006, 8(3):5 I4-525.

23. Holmes A], Bowyer J, Holley MP, O'Donoghue M, Montgomery M, Gillings MR: Diverse, yet-to-be-cultured members of the Rubrobacter subdivision of the Actinobacteria are widespread in Australian arid soils. FEMS Microbiol Ecol 2000, 33(2): I II-I 20.

24. Ferreira AC, Nobre MF, Moore E, Rainey FA, Battista JR, da Costa $M S:$ Characterization and radiation resistance of new isolates of Rubrobacter radiotolerans and Rubrobacter xylanophilus. Extremophiles 1999, 3(4):235-238.

25. Chen MY, Wu SH, Lin GH, Lu CP, Lin YT, Chang WC, Tsay SS: Rubrobacter taiwanensis sp. nov., a novel thermophilic, radiation-resistant species isolated from hot springs. Int J Syst Evol Microbiol 2004, 54(Pt 5): 1849-1855.

26. Arbiza L, Dopazo J, Dopazo H: Positive selection, relaxation, and acceleration in the evolution of the human and chimp genome. PLoS Comput Biol 2006, 2(4):e38.

27. Tajima F: Statistical method for testing the neutral mutation hypothesis by DNA polymorphism. Genetics 1989, I 23(3):585-595.

28. Yang Z, Bielawski JP: Statistical methods for detecting molecular adaptation. Trends Ecol Evol 2000, I 5( I 2):496-503.

29. Yang Z, Nielsen R: Estimating synonymous and nonsynonymous substitution rates under realistic evolutionary models. Mol Biol Evol 2000, 17(I):32-43.

30. Yang Z: PAML: a program package for phylogenetic analysis by maximum likelihood. Comput Appl Biosci 1997, I 3(5):555-556.

31. Wong WS, Yang Z, Goldman N, Nielsen R: Accuracy and power of statistical methods for detecting adaptive evolution in protein coding sequences and for identifying positively selected sites. Genetics 2004, 168(2): 104|-105I.

32. DnaSP [http://www.ub.es/dnasp/]

33. Zahradka K, Slade D, Bailone A, Sommer S, Averbeck D, Petranovic $M$, Lindner $A B$, Radman M: Reassembly of shattered chromosomes in Deinococcus radiodurans. Nature 2006 , 443(7III):569-573.

34. Potts M: Desiccation tolerance of prokaryotes. Microbiol Rev I 994, 58(4):755-805

35. Hua Y, Narumi I, Gao G, Tian B, Satoh K, Kitayama S, Shen B: Pprl: a general switch responsible for extreme radioresistance of Deinococcus radiodurans. Biochem Biophys Res Commun 2003, 306(2):354-360.

36. Narumi I, Satoh K, Cui S, Funayama T, Kitayama S, Watanabe H: PprA: a novel protein from Deinococcus radiodurans that stimulates DNA ligation. Mol Microbiol 2004, 54(I):278-285.

37. Petersen L, Bollback JP, Dimmic M, Hubisz M, Nielsen R: Genes under positive selection in Escherichia coli. Genome Res 2007, I7(9): 1336-1343.

38. Gutman PD, Fuchs P, Minton KW: Restoration of the DNA damage resistance of Deinococcus radiodurans DNA polymerase mutants by Escherichia coli DNA polymerase I and Klenow fragment. Mutat Res 1994, 3 I 4(1):87-97.

39. Database resources of the National Center for Biotechnology Information [ftp://ftp.ncbi.nih.gov/genomes/Bacteria]

40. MultiParanoid [http://www.sbc.su.se/ andale/multiparanoid/html/ index.html]

4l. BioEdit [http://www.mbio.ncsu.edu/BioEdit/bioedit.html]

42. Thompson JD, Higgins DG, Gibson TJ: CLUSTAL W: improving the sensitivity of progressive multiple sequence alignment through sequence weighting, position-specific gap penalties and weight matrix choice. Nucleic Acids Res 1994, 22(22):4673-4680.
43. Jukes TH, Cantor CR: Evolution of protein molecules. In Mammalian protein metabolism III Edited by: Munro HN. New York: Academic Press; 1969:21-132.

44. Carlson CS, Thomas DJ, Eberle MA, Swanson JE, Livingston RJ, Rieder MJ, Nickerson DA: Genomic regions exhibiting positive selection identified from dense genotype data. Genome Res 2005 , I5(I I): I553-1565
Publish with Biomed Central and every scientist can read your work free of charge

"BioMed Central will be the most significant development for disseminating the results of biomedical research in our lifetime. "

Sir Paul Nurse, Cancer Research UK

Your research papers will be:

- available free of charge to the entire biomedical community

- peer reviewed and published immediately upon acceptance

- cited in PubMed and archived on PubMed Central

- yours - you keep the copyright 